\title{
Reforming the Kidney Starting from a Single-Cell Suspension
}

\author{
Christodoulos Xinaris ${ }^{a}$ Takashi Yokoo ${ }^{b}$ \\ a IRCCS - Istituto di Ricerche Farmacologiche 'Mario Negri', Centro Anna Maria Astori, Science and Technology Park \\ Kilometro Rosso, Bergamo, Italy; ${ }^{b}$ Division of Nephrology and Hypertension, Department of Internal Medicine, \\ The Jikei University School of Medicine, Tokyo, Japan
}

\section{Key Words}

Single-cell suspension - Chronic kidney disease - Transplant

\begin{abstract}
Background: Chronic kidney disease affects $5-7 \%$ of people worldwide. The increasing number of patients and the shortage of transplantable organs create an imperative need to develop new methods for generating kidney tissue. Summary: Recent advances in our understanding of the developmental biology of the kidney, along with the establishment of novel methodologies in the field of regenerative medicine, have created significant potential for kidney regeneration. These advances incorporate both transplantation of metanephric primordia into adult recipients and construction of 'fetal' kidney tissue from suspensions of single cells of metanephric origin. This paper examines these approaches in the context of organ regeneration. Key Messages: The use of transplants of metanephric origin has the advantage over undifferentiated stem cells of already being committed to a renal developmental program. Although several technical difficulties remain to be overcome, the validation of these systems in preclinical models of renal disease will be of decisive importance in the coming years.
\end{abstract}

(c) 2014 S. Karger AG, Basel

\section{KARGER}

(c) 2014 S. Karger AG, Basel

$1660-2129 / 14 / 1262-0107 \$ 39.50 / 0$

E-Mail karger@karger.com

www.karger.com/nee

\section{Introduction}

While around 18,000 people with end-stage kidney disease receive a donor organ each year in the USA [1], there are more than 5 times as many patients on waiting lists [2]. The shortage of donor organs has increased the waiting time to over 3 years and the mortality rate by $5-10 \%$. Owing to these problems, novel strategies are increasingly being called for and multiple approaches are being pursued. Transplantation of developing fetal kidney tissues from animal embryos so that they will grow and mature in situ in the recipient provides significant advantages. Developing metanephric tissue is less immunogenic than the adult kidney because it contains fewer antigen-presenting cells and expresses fewer MHC class I and class II antigens that mediate host recognition [3]. Compared to embryonic stem cells or induced pluripotent stem cells, metanephric cells are already committed to a genetic program of renal development, obviating the need to preprogram the cell fate and thus minimizing the need to engineer a complete organ.

During the last decade, studies have shown that it is possible to grow new kidneys in adult living hosts or integrate new filtering nephrons into immature kidneys. Em- 
bryonic kidney rudiments implanted beneath the renal capsule $[4,5]$, within the omentum $[5,6]$ or into tunnels in the cortices of host kidneys [7] become integrated into living animals. Cultured fetal kidneys [8] also become vascularized and form mature glomeruli upon in vivo transplantation. Kidney-like tissues can be generated via recombination of in vitro propagated ureteric buds (UB) with intact metanephric mesenchyme (MM) $[9,10]$. These tissues undergo early vascularization and yield morphologically normal glomeruli upon in vivo implantation.

Suspensions of single cells can be used to produce kidney tissue. Bioengineering studies have shown that metanephric cells strongly expressing Sall- 1 can generate in vitro a $3 \mathrm{D}$ renal tissue containing rudimental glomerularlike structures [11]. Advances in developmental biology have shown that a simple suspension of cells isolated from murine metanephros can self-organize in vitro into a 'fetal tissue' that contains immature nephrons and collecting ducts [12]. A strategic step forward was recently made with the demonstration that organoids constructed in vitro from suspensions of metanephric cells can be integrated into a living recipient to form vascularized glomeruli and to exert kidney-specific functions [13].

All of these advances, with a specific focus on the transplantation of metanephric primordia and the construction of tissues from cell suspensions, will be discussed in this paper.

\section{Metanephros Transplantation as a Source of Organ Regeneration}

The metanephros is used in basic research studies and holds promise as a potential source for the successful regeneration of a functional whole kidney. A metanephros transplanted into a host renal cortex may continue to grow into mature tissues that contain functional tubuli and vascularized glomeruli endowed with a filtering capacity [7]. A metanephros has also been transplanted into a host omentum. The transplanted tissue in the omentum produced urine and, after an intact ureteroureterostomy with the ureter of the native kidney that was removed, anephric rats started to void and showed prolonged life spans [5]. Furthermore, metanephroi from porcine embryos implanted either in the omentum of mice in which costimulation was blocked [5] or under the kidney capsules of immunodeficient mice [4] developed fully functional nephrons. The average levels of urea nitrogen and creatinine were higher in the cyst fluid that arose from the transplanted tissue than in the sera of the transplanted mice [4]. This also showed that metanephros transplantation can maintain the blood pressure in anephric rats with induced acute hypotension [14] and reduce vascular calcification in rats with chronic renal failure [15], suggesting that transplanted metanephroi have multiple renal functions other than urine production. Although the omentum is used mainly because a tight renal capsule does not confine it, and the host's vessels can be preferentially integrated into the transplant, the low hydrostatic pressure in the omentum may affect the acquisition of some renal function in the developed metanephros. In fact, Matsumoto et al. [16] revealed that the para-aortic area is better for the establishment of renin-producing tissue. These results suggest a rationale for the usefulness of the metanephros as a potential source of transplantable regenerated kidney to address the shortage of organs for kidney transplantation.

Matsumoto et al. [6] recently showed that xenotransplanted metanephros could supply endogenous mesenchymal stem cells with a niche for differentiation into renal tissue. PCR using species-specific primers and sequence analysis revealed that xenotransplanted metanephros, from rat to mouse and similarly from pig to cat, expresses erythropoietin (EPO) of host animal origin. This revealed that the EPO-producing cells came from the host animals and could develop to produce EPO in the transplanted metanephros. It was further shown that the EPO-producing cells did not originate from integrating vessels but rather from circulating host mesenchymal stem cells mobilized from bone marrow.

In addition, conventional metanephros transplantation should require continuous and strong immunosuppression to avoid humoral rejection that occurs across the xenogeneic barrier, which evokes various adverse effects including carcinogenicity and severe rejection. Therefore, for safety, when the xenotransplant is no longer needed it should be discarded by introducing a cell fateregulating system in which a suicide gene is expressed on demand. In this context, E2F1 was suited for this purpose. $\mathrm{E} 2 \mathrm{~F} 1$ is a transcription factor which regulates cell proliferation, and its ectopic expression induces apoptosis. Therefore, transgenic mice expressing E2F1 under the control of tamoxifen (ER-E2F1 mice) were generated. The administration per os of tamoxifen in host rats wherein metanephroi of ER-E2F1 mice were transplanted activated the suicide gene, and the xenocompartments of transplanted metanephroi were cleared by apoptosis, leaving the autologous EPO-producing tissues [6]. It is also confirmed that established transplants no longer need immunosuppressants while maintaining EPO pro- 
duction [6]. These results indicate that xenometanephroi per se could acquire some renal function in the host omentum but also supply a niche for host stem cells to regenerate renal tissues and then be rebuilt to constitute only host cell components. These data may also help to reduce the adverse effects of long-term immunosuppressant administration and to moderate the ethical issues surrounding xenotransplantation.

\section{Generation of Kidney Tissue from Metanephric Progenitor Cells}

One of the most promising tools in mammalian kidney engineering is the generation of fetal or mature tissues by making use of the intrinsic proficiency of cells to organize themselves into fully self-derived sophisticated structures $[17,18]$. Some of the earliest descriptions of this strategy demonstrated that renal epithelial cell lines cultured in extracellular matrix gels could be induced to create branching tubules either by the whole embryonic kidney or by the MM [reviewed in 19]. Advancement of this methodology showed that UB cells that were isolated from mice at embryonic day 11.5 (E11.5) and propagated in vitro were able to undergo branching tubulogenesis when stimulated by a conditioned medium from an MM-derived cell line [20]. Similarly, a tubular epithelial cell line or mesenchymal stem cells created glomerular and tubular structures in subcutaneous spaces when stimulated by a combination of media from vascular endothelial and tubular cells [21]. These studies suggest that simple cultures of cells derived from undifferentiated MM and unbranched UB could be employed to create branching tubular structures similar to those that form in normal metanephros.

Bioengineering attempts by Osafune et al. [11] showed that suspensions of murine metanephric mesenchymal E11.5 progenitor cells selected for a high expression of Sall-1-a zinc-finger nuclear factor - which is essential during kidney development - can generate in vitro 3D renal epithelia which contain glomerular-like structures. This cell preparation required coculture with an exogenous spinal cord cell layer, as has been done in traditional developmental studies [22]. Even though the functional capacity of these structures has not been studied in vivo, this approach suggests that dispersed cells in suspension can be used as starting material to obtain rudimental 3D kidney tissues. More progress in the field was made with the development of a method that allows the generation of 'fetal' kidney tissue from suspensions of E11.5 kidney cells without the use of any exogenous tissue

Reforming the Kidney Starting with a

Single-Cell Suspension
[12]. Although this method reproduced the anatomies and differentiation states of nephrons and stroma successfully, collecting ducts developed as a multitude of very small collecting duct trees rather than a continuous one as would happen in a normal kidney. An improved version of this system, combining a single reaggregated UB with reaggregated mesenchyme, allowed the formation of nephrons arranged around a single collecting duct tree [23]. These attempts, however, failed to develop glomeruli to any meaningful extent since the avascular in vitro environment does not allow complete glomerulogenesis. This impediment places a limitation on the maturation potential of tissue obtained from a simple culture or suspension of precursor cells. Thus, the capacity of these cells to generate nephrons with functional properties remained unknown until recently.

Starting with simple suspensions of single kidney cells, Xinaris et al. [13] finally constructed 'organoids' that could carry out kidney functions when implanted into a living animal, including glomerular filtration, the tubular reabsorption of macromolecules, and the production of EPO. These organoids were obtained from single-cell suspensions derived from E11.5 murine kidneys and then implanted beneath the kidney capsule of an athymic rat host (fig. 1). The key maneuver to obtain complete glomerulogenesis consisted of soaking the organoids in a solution containing vascular endothelial growth factor (VEGF) and then injecting VEGF into the recipient animals after the organoids had been implanted in the kidney. This method of implantation restituted the efficacy of the podocyte-endothelial VEGF axis that is required for glomerular capillary endothelial induction and resulted in the formation of vascularized glomeruli with fully differentiated glomerular capillary walls, including endothelial fenestrae, foot processes, and slit diaphragms [13] (fig. 2). These organoids were able to develop glomeruli with normal-appearing slits in vivo, wherein the proportion of diaphragms was comparable to that found in the adult glomerulus [unpubl. data]. When the ultrafiltering function of the intragraft nephrons was tested by injecting fluorescent dextrans of increasingly high molecular weight into the host blood system, the proximal tubule cells concentrated dextrans only of lower molecular weights from the lumen, implying efficient ultrafiltration.

Approaches starting from the metanephric kidney and dissociating down to the single-cell level provide a valuable window for performing pharmacologic or genetic modifications in individual cells prior to the in vitro aggregation step $[13,24]$. For instance, the single-cell stage allows the 


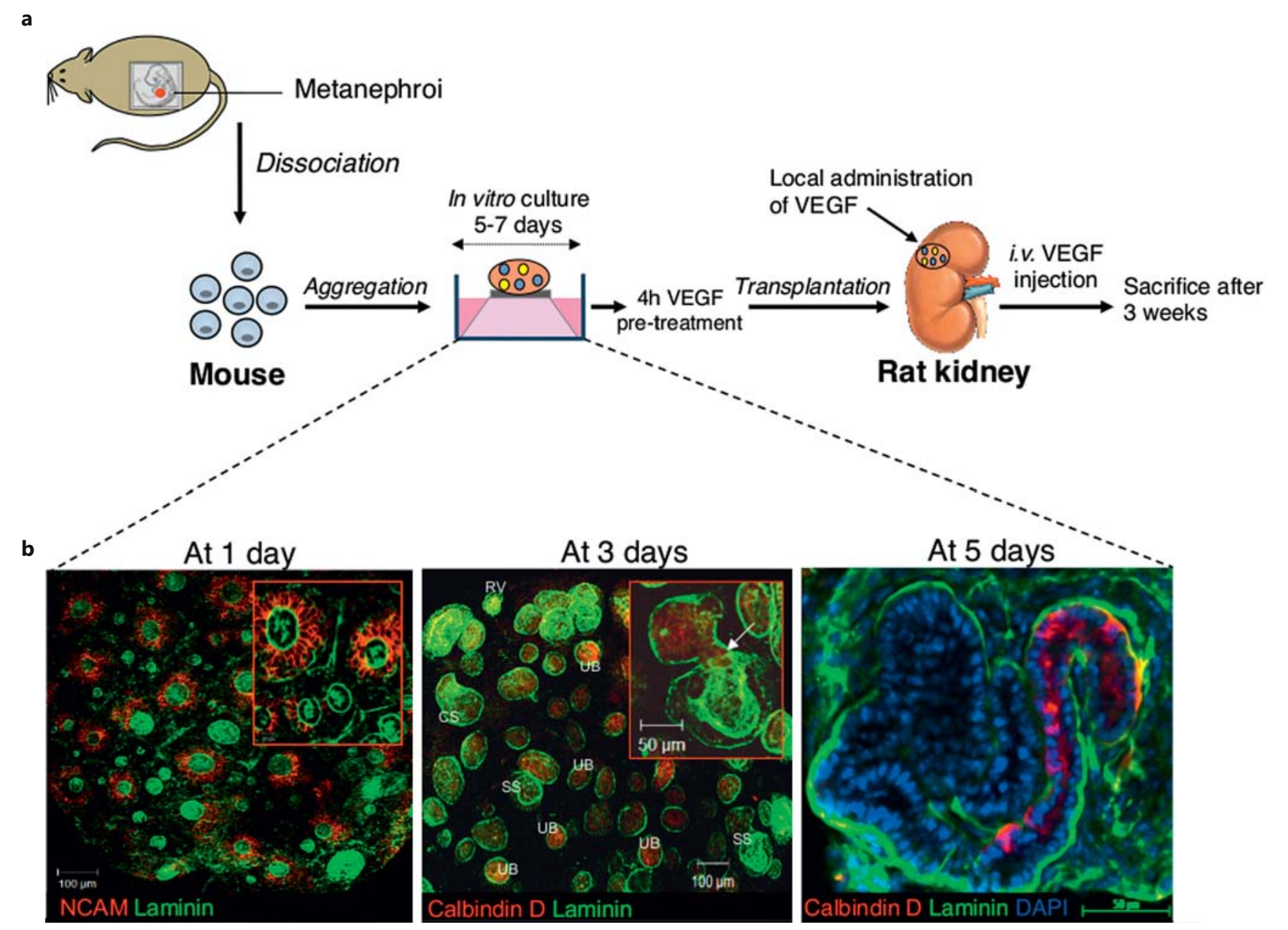

Fig. 1. Experimental design. a E11.5 mouse embryonic kidneys were fully dissociated into single cells. On suspension, the cells were recollected by centrifugation and the pellets were cultured in vitro on a filter on a metal grid. After 5 days, the aggregates (organoids) were pretreated with $2 \mu \mathrm{g}$ VEGF and implanted beneath the renal capsule of athymic rats subjected to right nephrectomy. Rat kidneys were treated locally with $1 \mu \mathrm{g}$ VEGF immediately after renal organoid implantation, and recipients received intravenous injections of
VEGF for 3 weeks. b At 1 day, NCAM (red) was strongly expressed by condensed MM around the UB tips. After 3 days, the organoid developed laminin-positive epithelial renal structures (green), including renal vesicles (RV), comma-shaped bodies (CS), and Sshaped bodies (SS). The developing nephron showed a connection to the UB (arrow in the inset). At 5 days, elongating tubular profiles also became visible in continuity with calbindin D28k-positive epithelia (red). Adapted from Xinaris et al. [13]. use of genetic engineering approaches to 'humanize' cells, possibly by introducing immunomodulatory genes or by switching off others, to reduce the possibility of rejection and facilitate the xenotranplantation of animal renal tissues [24]. Self-forming kidneys can be also exploited to generate human renal tissue by first constructing chimeric kidneys that combine animal progenitor cells and human stem cells, followed by the selective elimination of animal cells after transplantation. For example, the organoids have been shown to incorporate cells of another source, such as human amniotic fluid stem cells [25] or induced pluripotent stem cell-derived OSR1-positive cells [26], to build chimeric tissue in vitro. Growing and transplanting such human chimeras by using this technology may facilitate more focused developmental studies and greatly augment the potential for modeling of kidney diseases and cellbased therapeutic strategies. 
Fig. 2. In vivo maturation of organoids formed from embryonic cell suspensions. Macroscopic examination showed greatly enhanced vascularization of the graft (arrows) in VEGF-treated (a) compared to VEGF-untreated recipients (b) 3 weeks after implantation. c Histology showed several glomerular profiles (arrows; HE) with glomerular arterioles (arrowheads in the inset). d Glomerular structures contained red cells (arrows in the inset; HE). e A glomerular tuft showed capillary mouse endothelial cell antigen-32 staining in an organoid taken after engraftment and VEGF treatment (3 weeks). f, g Electron micrographs of intragraft glomerular structures. f A capillary structure containing erythrocytes (Er) was lined by endothelium (EC). g Complete foot processes (fp) and slit diaphragms (SD; arrows) tightly separated the glomerular basement membrane (GBM) from the urinary space (US). The endothelium showed fenestration (arrows). $\mathrm{P}=$ Podocyte. Adapted from Xinaris et al. [13].
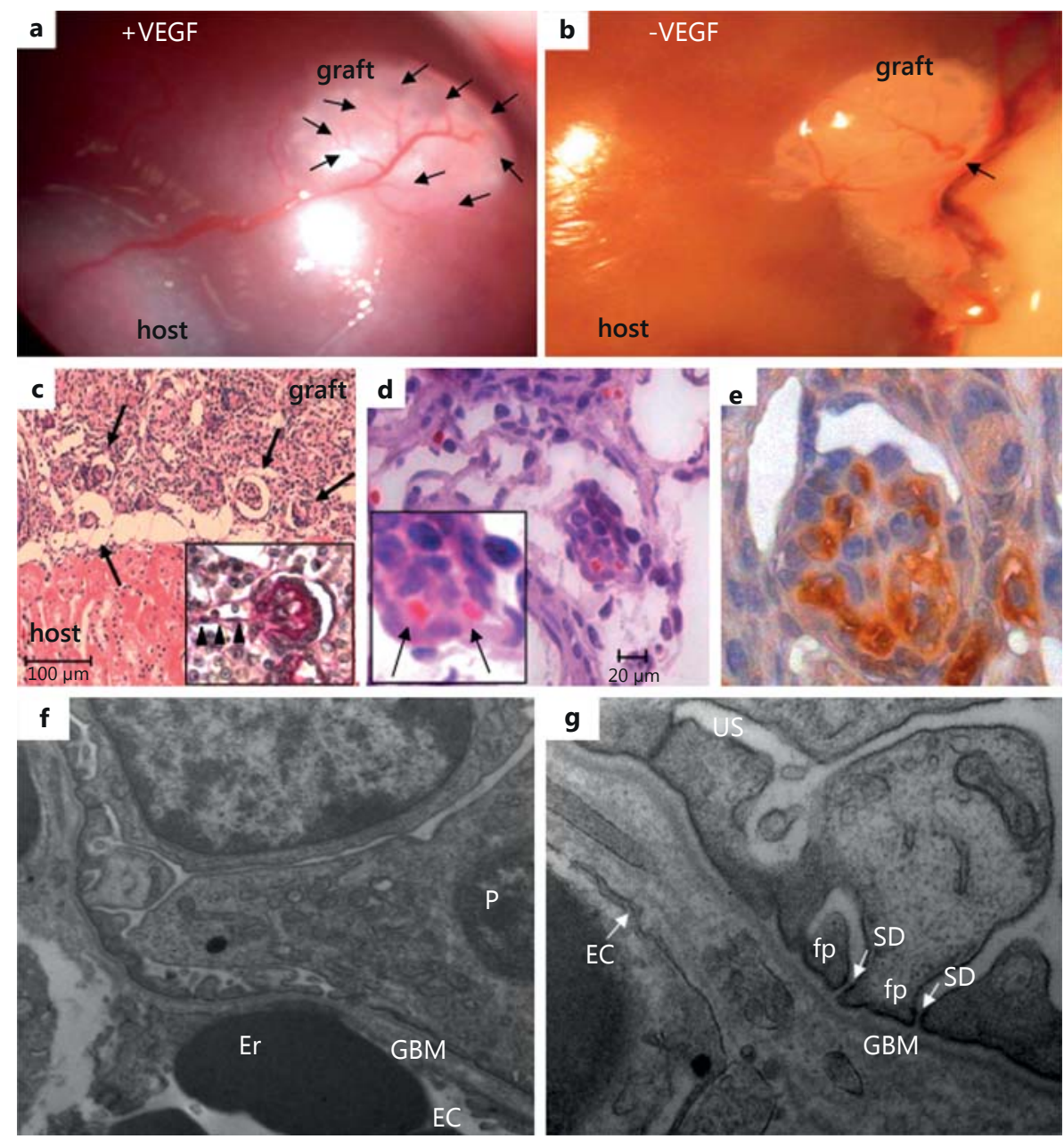

\section{Conclusions}

New frontiers are being explored in the field of regenerative nephrology. A number of emerging approaches take advantage of the existing developmental programs to grow new functional kidneys within living recipients. These systems involve the transplantation of metanephric primordia into adult recipients, as well as the construction of kidneys or kidney-like tissues from simple suspensions of metanephric cells. The use of transplants of metanephric origin has the advantage over undifferentiated stem cells of already being committed to perfectly executing a renal developmental program, thus obviating the need for direct differentiation and organ morphogenesis. In order for the transplantation of metanephric rudimental tissues to be clinically applicable, a number of challenges must be overcome: first, transplanted tissues must develop into 3D organs with the shape, volume, and anatomy of human kidneys, able to sustain life long term; second, developing transplants need to become fully integrated into the host in terms of inducing a state of minimal immune response and being vascularized by host vessels, and finally, the nascent nephrons should be linked to a draining collecting system. Despite these challenges, the strategies described herein open a novel direction for generating donor tissue suitable for transplantation. Evaluation of these systems within preclinical models of renal disease will be of significant importance in the coming years.

\section{Acknowledgements}

We thank Dr. Susanna Tomasoni and Dr. Mauro Abbate for their valuable comments. The authors also thank Antonella Piccinelli for her contribution to the figures, and Manuela Passera for her technical assistance. 


\section{References}

1 Centers for Disease Control and Prevention: National Chronic Kidney Disease Fact Sheet: General Information and National Estimates on Chronic Kidney Disease in the United States, 2010. Atlanta, US Department of Health and Human Services, Centers for Disease Control and Prevention, 2010.

2 US Department of Health and Human Services: National Transplantation Data Report. Richmond, Organ Procurement and Transplantation Network, 2013.

$\checkmark 3$ Hammerman MR: Growing new kidneys in situ. Clin Exp Nephrol 2004;8:169-177.

$\checkmark 4$ Dekel B, Burakova T, Arditti FD, et al: Human and porcine early kidney precursors as a new source for transplantation. Nat Med 2003;9: 53-60.

$>5$ Rogers SA, Lowell JA, Hammerman NA, Hammerman MR: Transplantation of developing metanephroi into adult rats. Kidney Int 1998;54:27-37.

$\checkmark 6$ Matsumoto K, Yokoo T, Matsunari H, et al: Xenotransplanted embryonic kidney provides a niche for endogenous mesenchymal stem cell differentiation into erythropoietinproducing tissue. Stem Cells 2012;30:12281235.

7 Woolf AS, Hornbruch A, Fine LG: Integration of new embryonic nephrons into the kidney. Am J Kidney Dis 1991;17:611-614.

$>8$ Robert B, St John PL, Hyink DP, Abrahamson DR: Evidence that embryonic kidney cells expressing flk-1 are intrinsic, vasculogenic angioblasts. Am J Physiol 1996;271:F744-F753.

$>9$ Rosines E, Sampogna RV, Johkura K, et al: Staged in vitro reconstitution and implantation of engineered rat kidney tissue. Proc Natl Acad Sci USA 2007;104:20938-20943.
10 Steer DL, Bush KT, Meyer TN, Schwesinger C, Nigam SK: A strategy for in vitro propagation of rat nephrons. Kidney Int 2002;62: 1958-1965.

-11 Osafune K, Takasato M, Kispert A, Asashima M, Nishinakamura R: Identification of multipotent progenitors in the embryonic mouse kidney by a novel colony-forming assay. Development 2006;133:151-161.

12 Unbekandt M, Davies JA: Dissociation of embryonic kidneys followed by reaggregation allows the formation of renal tissues. Kidney Int 2010;77:407-416.

13 Xinaris C, Benedetti V, Rizzo P, et al: In vivo maturation of functional renal organoids formed from embryonic cell suspensions. J Am Soc Nephrol 2012;23:1857-1868.

14 Yokote S, Yokoo T, Matsumoto K, et al: The effect of metanephros transplantation on blood pressure in anephric rats with induced acute hypotension. Nephrol Dial Transplant 2012;27:3449-3455.

15 Yokote S, Yokoo T, Matsumoto K, et al: Metanephros transplantation inhibits the progression of vascular calcification in rats with adenine-induced renal failure. Nephron Exp Nephrol 2012;120:e32-e40.

16 Matsumoto K, Yokoo T, Yokote S, et al: Functional development of a transplanted embryonic kidney: effect of transplantation site. J Nephrol 2012;25:50-55.

17 Suga H, Kadoshima T, Minaguchi M, et al: Self-formation of functional adenohypophysis in three-dimensional culture. Nature 2011; 480:57-62.
18 Eiraku M, Takata N, Ishibashi H, et al: Selforganizing optic-cup morphogenesis in three-dimensional culture. Nature 2011;472: 51-56.

19 Steer DL, Nigam SK: Developmental approaches to kidney tissue engineering. Am J Physiol Renal Physiol 2004;286:F1-F7.

20 Sakurai H, Barros EJ, Tsukamoto T, Barasch J, Nigam SK: An in vitro tubulogenesis system using cell lines derived from the embryonic kidney shows dependence on multiple soluble growth factors. Proc Natl Acad Sci USA 1997; 94:6279-6284.

21 Machiguchi T, Nakamura T: Cellular interactions via conditioned media induce in vivo nephron generation from tubular epithelial cells or mesenchymal stem cells. Biochem Biophys Res Commun 2013;435:327-333.

22 Auerbach R, Grobstein C: Inductive interaction of embryonic tissues after dissociation and reaggregation. Exp Cell Res 1958;15:384397.

23 Ganeva V, Unbekandt M, Davies JA: An improved kidney dissociation and reaggregation culture system results in nephrons arranged organotypically around a single collecting duct system. Organogenesis 2011;7:83-87.

24 D’Agati VD: Growing new kidneys from embryonic cell suspensions: fantasy or reality? J Am Soc Nephrol 2012;23:1763-1766.

25 Siegel N, Rosner M, Unbekandt M, et al: Contribution of human amniotic fluid stem cells to renal tissue formation depends on mTOR. Hum Mol Genet 200;19:3320-3331.

26 Mae S, Shono A, Shiota F, et al: Monitoring and robust induction of nephrogenic intermediate mesoderm from human pluripotent stem cells. Nat Commun 2013;4:1367. 